\title{
Structural Studies of Predicted Ligand Binding Sites and Molecular Docking Analysis of Slc2a4 as a Therapeutic Target for the Treatment of Cancer
}

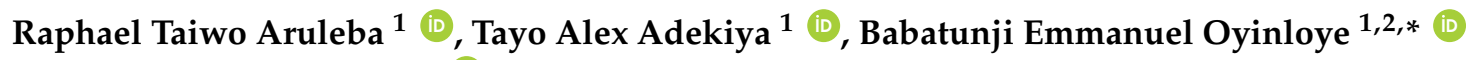 \\ and Abidemi Paul Kappo ${ }^{1}$ \\ 1 Biotechnology and Structural Biochemistry (BSB) Group, Department of Biochemistry and Microbiology, \\ University of Zululand, KwaDlangezwa 3886, South Africa; arulebataiwo@yahoo.com (R.T.A.); \\ adekiyatalex@gmail.com (T.A.A.); KappoA@unizulu.ac.za (A.P.K.) \\ 2 Department of Biochemistry, Afe Babalola University, PMB 5454, Ado-Ekiti 360001, Nigeria \\ * Correspondence: babatunjioe@abuad.edu.ng; Tel.: +27-71-099-5087; Fax: +27-35-902-6567
}

Received: 4 November 2017; Accepted: 28 November 2017; Published: 28 January 2018

\begin{abstract}
Presently, many studies have focused on exploring in silico approaches in the identification and development of alternative therapy for the treatment and management of cancer. Solute carrier family-2-member-4-gene (Slc2a4) which encodes glucose transporter 4 protein (GLUT4), has been identified as a promising therapeutic target for cancer. Though Slc2a4 is known to play a major regulatory role in the pathophysiology of type 2 diabetes, emerging evidence suggests that successful pharmacological inhibition of this protein may lead to the development of a novel drug candidate for the treatment of cancer. In this study, Slc2a4 protein sequence was retrieved and analysed using in silico approaches, and we identified seven putative antimicrobial peptides (AMPs; RAB1-RAB7) as anti-cancer. The structures of the protein and AMPs were modelled using I-TASSER server, and the overall quality of the Slc2a4 model was validated using PROCHECK. Subsequently, the probable motifs and active site of the protein were forecasted. Also, the molecular interaction between the AMPs and Slc2a4 was ascertained using PatchDock. The result revealed that, all the AMPs are good Slc2a4 inhibitors with RAB1 having the highest binding affinity of 12,392 and binding energy of $-39.13 \mathrm{kcal} / \mathrm{mol}$. Hence, this study reveals that all the generated AMPs can serve as therapeutic drug in treating cancer by inhibiting Slc2a4 which is responsible for the production of energy for cancer cells during angiogenesis. This is the first report on AMPs as inhibitors of Slc2a4 for the treatment of cancer.
\end{abstract}

Keywords: antimicrobial peptides; cancer; docking; homology modelling; Slc2a4

\section{Introduction}

Over the last several decades, there has been a fundamental shift in the global disease burden from infectious diseases to cancer and other non-communicable diseases [1]. Cancer is a group of multifactorial disease caused by the uncontrolled growth and spread of abnormal cells in the body. Cancer cells are characterized by their ability to rapidly grow and divide, and undergo uncontrolled proliferation [2,3]. Cancer is a major public health issue and has been implicated as a global menace exacerbated by environmental and lifestyle factors [4]. It is believed that this group of diseases are associated with both genetic and metabolic abnormalities [5]. However, accumulating evidence indicates that cancer is predominantly a metabolic disease. This fact is in agreement with the original theory of Otto Warburg [6].

The burden of this disease is predicted to increase worldwide due to recent advancement and aging of the population, mainly in the developing countries, where about $82 \%$ of the world's population 
resides [7]. According to GLOBOCAN estimates in 2012, about 14.1 million new cancer cases were diagnosed in 2012, with cancer accounting for about 8.2 million deaths globally in the same year. Except preventive and adequate management measures are put in place, global cancer death rate has been projected to rise to about 14.6 million annually by 2035 [7-9]. Although great strides with significant results have been made in the treatment and control of cancer progression, noteworthy severe side effects on normal tissues and organs combined with various deficiencies of the current chemotherapeutic drugs used in the treatment cancer calls for urgent attention and development of an alternative therapy for the treatment and control of cancer $[10,11]$.

It has been reported in literature that due to the requirement of energy to feed and maintain survival of uncontrolled proliferation, cancer cells consume more glucose than their normal counterparts [2]. This unique attribute of cancer cells (elevated glucose utilization) is been exploited for the development of novel therapy and diagnostic tools for cancers. Assessment of glucose entry into tum our cells establishes glucose uptake to be a key rate-limiting step in glucose metabolism, suggesting the efficacy of targeting glucose transport for therapy [12]. Emerging evidence suggests that pharmacological inhibition of Solute carrier family-2-member-4-gene (Slc2a4), which encodes glucose transporter 4 protein (GLUT4), is an attractive therapeutic target for the development of a novel drug candidate for the treatment of cancer [12-14]. Members of this family have tissue specific expression, biochemical properties and physiologic functions that operate together to regulate and maintain glucose levels and distribution. Slc2a4 is an insulin-sensitive glucose transporter known to play an essential role in glucose homeostasis [15].

It has been established that increased cellular glucose uptake and metabolism is a vital requirement for rapid proliferation in tumorigenesis [14]. GLUT4 demonstrates a unique relationship with cancer in a manner comparable to that of GLUT1, since both transporters are transcriptionally repressed by p53, a known tumour suppressor protein that plays an essential role in cell cycle control and apoptosis [16]. Any alteration or mutation within the DNA-binding domain of p53 will possibly lead to dysregulation or overexpression of GLUT4 in certain types of cancer. This important factor makes this protein an attractive target in treatment of cancer $[14,16]$.

Antimicrobial peptides (AMPs) are currently been explored as an essential source for the development of new therapeutic drugs due to their multifunctional properties. They can act as drug delivery vector, signalling molecule, immunomodulatory agent, and mitogenic and antitumour agent [17]. This study using in silico approach sought to identify potential plant antimicrobial peptides as selective inhibitors of Slc2a4 in order to develop a more potent anti-cancer therapy with high efficacy, excellent tolerability, and few transient side effects.

\section{Results}

\subsection{Physicochemical Properties and the Abundance of Amino Acids in Slc2a4 Protein}

The ExPASy result indicated that Slc2a4 protein sequence comprises of 509 amino acid residues with all the 20 amino acids (Figure 1) contributing to give the protein an average molecular weight of $54.8 \mathrm{kDa}$. Additionally, Figure 1 indicated that the most abundant amino acids in Slc2a4 is leucine with $15.5 \%$, followed by glycine, alanine, and valine with the percentage of $10 \%, 9.2 \%$, and $7.9 \%$, respectively. Histidine and cysteine contributed the lowest abundance residues with $0.6 \%$ and $0.8 \%$ respectively, followed by tryptophan, lysine, and aspartate, which contributed $1.4 \%, 1.6 \%$, and $1.8 \%$ amino acid residue to $S l c 2 a 4$, respectively.

In Table 1, the physicochemical parameters predicted the protein to be a neutrally charged protein as a result of equal numbers of positively charged residues (arginine $4.9 \%$ and lysine $1.6 \%$ ) and negatively charged residues (aspartic acid $1.8 \%$ and glutamic acids $4.7 \%$ ), respectively. These four amino acids are the major contributors to the overall charge of a protein because at neutral $\mathrm{pH}$, arginine and lysine are positively charged, while aspartate and glutamate are negatively charged [18]. 


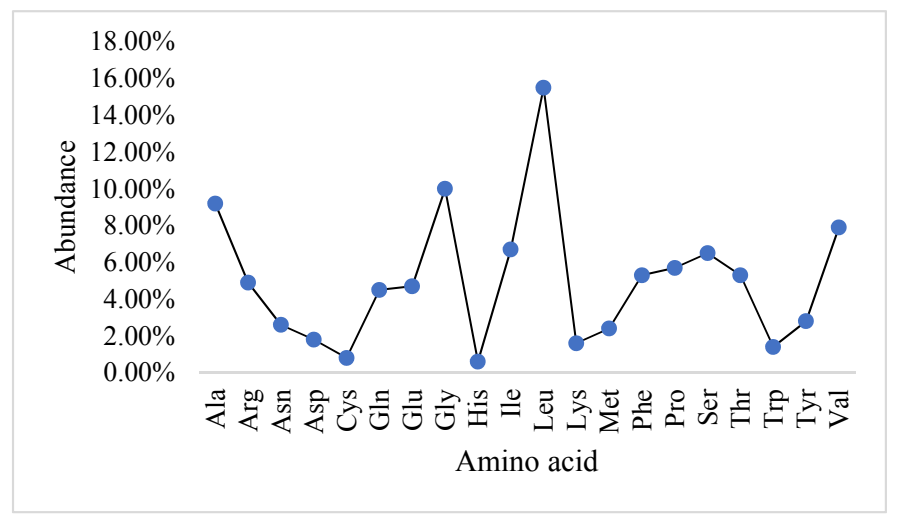

Figure 1. Graphical representation of the abundance of 20 amino acid presents in Slc2a4. Leucine has the highest abundance, and histidine has the lowest abundance.

Table 1. Physicochemical parameters of Slc2a4.

\begin{tabular}{|c|c|}
\hline ProtParam Parameters & Values \\
\hline Number of amino acids & 509 \\
\hline Molecular weight & $54,895.50 \mathrm{Da}$ \\
\hline Theoretical Pi & 6.86 \\
\hline Atomic composition & $\begin{array}{c}\text { Carbon C: } 2530 \\
\text { Hydrogen H: } 4009 \\
\text { Nitrogen N: } 641 \\
\text { Oxygen O: } 686 \\
\text { Sulfur S: } 16\end{array}$ \\
\hline Formula & $\mathrm{C}_{2530} \mathrm{H}_{4009} \mathrm{~N}_{641} \mathrm{O}_{686} \mathrm{~S}_{16}$ \\
\hline Number of negatively charged residues & 33 \\
\hline Number of positively charged residues & 33 \\
\hline Extinction coefficient & $\begin{array}{c}59,610 \\
\text { Abs } 0.1 \%(=1 \mathrm{~g} / \mathrm{L}) 1.086 \text {, assuming all pairs of Cys residues form cystines } \\
59,360 \\
\text { Abs } 0.1 \%(=1 \mathrm{~g} / \mathrm{L}) 1.081 \text {, assuming all Cys residues are reduced }\end{array}$ \\
\hline Total number of atoms & 7882 \\
\hline Estimated Half-life & $\begin{array}{l}30 \mathrm{~h} \text { (mammalian reticulocytes, in vitro). } \\
>20 \mathrm{~h} \text { (yeast, in vivo). } \\
>10 \mathrm{~h} \text { (Escherichia coli, in vivo). }\end{array}$ \\
\hline Aliphatic index & 118.61 \\
\hline Grand average of hydropathicity (GRAVY) & 0.556 \\
\hline Instability index & 39.83 \\
\hline
\end{tabular}

Moreover, with 2530 Carbon $(\mathrm{C}), 4009$ Hydrogen $(\mathrm{H}), 641$ Nitrogen $(\mathrm{N})$, and 686 Oxygen $(\mathrm{O})$, the atomic composition of Slc2a4 adds up to 7882. Also, the protein is acidic as indicated by the isoelectric point which was 6.86. The estimated half-life of this protein shows that the protein can remain intact without being degraded for $30 \mathrm{~h}$ in human, less than $20 \mathrm{~h}$ in yeast and less than $10 \mathrm{~h}$ in E. coli. The extinction coefficient which describes the quantity of light a protein can absorb at a specific wavelength was computed using tyrosine, tryptophan and cysteine. The generated aliphatic index was 118.61, with 0.556 GRAVY and instability index of 39.83 .

\subsection{Prediction of $2 D$-Structure of Slc2a4 Protein}

As presented in Figure 2, the predicted result by PsiPred server revealed that the secondary structure of Slc $2 a 4$ contains $22 \alpha$-helices, $6 \beta$-strands, and 28 coils. The high number of $\alpha$-helices in the 
predicted 2D structure confirmed that $S l c 2 a 4$ is a transmembrane protein, capable of mediating the transportation of glucose across the cell membrane [19-21]. A lot of transmembrane proteins comprise exclusively of $\alpha$-helices that are available in the cytoplasmic membrane region, while some membrane proteins do have $\beta$-strands [22].

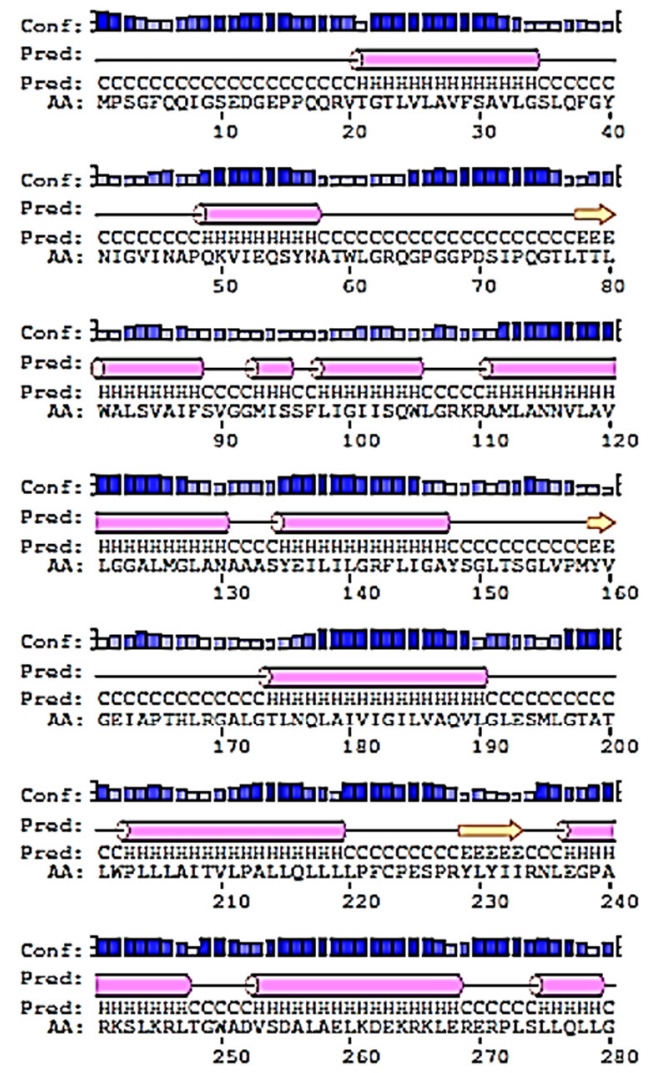

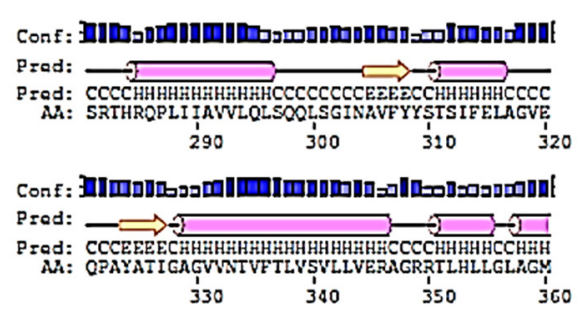

Conf : Znس

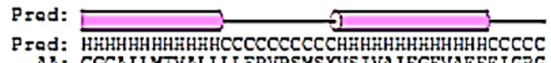

rad: HHHHHHHHНHAHCCCCCCCCCCHHHHHHHHHHHHHCCCCC

$\begin{array}{cccc}1 & 1 & 1 & 1 \\ 370 & 390 & 390 & 400\end{array}$

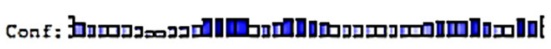

Prad:

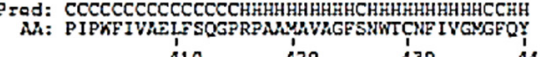

$\begin{array}{llll}410 & 420 & 430 & 440\end{array}$

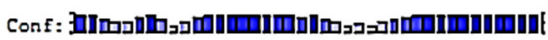

Prad:

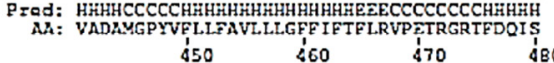

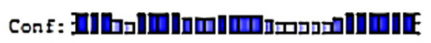

Prad: Prad: HHHeccecececcecceccecceccec

Prad: HHHHCCCCCCCCCCCCCCCCCCCCCCCCC $\begin{array}{ll}190 & 500\end{array}$

\begin{tabular}{|c|c|c|}
\hline LAgand: & & \\
\hline$\stackrel{\square}{\longrightarrow}$ & $\begin{array}{l}\text { - nel1x } \\
\text { - gtrans }\end{array}$ & $\begin{array}{l}\text { cont: balll - contreance ot prodiction } \\
\text { wred: prealteted seconeary setucture }\end{array}$ \\
\hline
\end{tabular}

Figure 2. Secondary structure prediction of Slc2a4 using PSIPRED. Slc2a4 is predicted to consist of $22 \alpha$-helices and $6 \beta$-strands.

\subsection{Discovery of Putative AMPs and Physicochemical Characterization}

Antimicrobial Peptide Databases (CAMP and DRAMP) were visited to retrieve experimentally validated anticancer AMPs. It was revealed that CAMP and DRAMP had 28 and 277, respectively, which are experimentally validated, interred, and synthetic anticancer AMPs. Only 25 anticancer AMPs that have been experimentally validated from plants origin were identified and retrieved. Moreover, the retrieved data was suggested to further analysis in order to remove the experimentally validated anticancer AMPs that are duplicated, and finally only 13 AMPs were generated.

The HMMER multiple module was used in the construction of the AMPs profile, and the ENSEMBL server (http://www.ensembl.org/index.html) and UNIPROT database (http://www. uniprot.org/) were visited to retrieve at least a thousand genome sequences in FASTA format. In order to identify the putative anti-cancer AMPs, the constructed profile was queried against all the genome sequences with an E-value cut-off of 0.01. Seven (7) peptides were identified which were all considered to be putative anticancer AMPs and all identified AMPs were ranked in accordance to their E-values, starting from the lowest to the highest.

Thereafter, the physicochemical properties of the seven generated best putative anticancer AMPs were determined to ascertain the characteristics embedded in them for further usage in interaction study with Slc2a4. As shown in Table 2, it was discovered that none of the putative AMPs has $100 \%$ similarity with the occurring anticancer AMPs or experimentally validated AMPs, which indicated that 
all the seven identified sequence of the putative AMPs were novel. Also, it was discovered that out of the seven putative anticancer AMPs generated, two of the AMPs had positive net charge and another two of the AMPs has net negative charge, while three has zero net charge; this is zero net charge may be due to the low or absent of positively charged amino acids in the AMP sequence. Furthermore, the physicochemical analysis of the putative peptide indicates that most of the peptide contain higher amount of cysteine residues, which indicated that the homologue 3D model of the peptides will be properly folded, and this will also contribute to the binding affinity of the peptides to the Slc2a4.

Table 2. Physicochemical properties for the seven putative anti-cancer antimicrobial peptides (AMPs).

\begin{tabular}{ccccccccc}
\hline $\begin{array}{c}\text { Putative } \\
\text { AMPs }\end{array}$ & $\begin{array}{c}\text { Mass } \\
\text { (Da) }\end{array}$ & $\begin{array}{c}\text { Most } \\
\text { Common } \\
\text { Amino Acids }\end{array}$ & $\begin{array}{c}\text { Isoelectric } \\
\text { Point }\end{array}$ & $\begin{array}{c}\text { Net } \\
\text { Charge }\end{array}$ & $\begin{array}{c}\text { Total } \\
\text { Hydrophobic } \\
\text { Ratio (\%) }\end{array}$ & $\begin{array}{c}\text { Bosman } \\
\text { Index } \\
\text { (kcal/mol) }\end{array}$ & $\begin{array}{c}\text { Half-Life } \\
\text { (h) }\end{array}$ & $\begin{array}{c}\text { Sequence Similarity } \\
\text { with Other Molecules } \\
\text { and Percentage (\%) }\end{array}$ \\
\hline RAB 1 & 3038.03 & Cys: 20.69 & 6.03 & 0 & 51 & 0.18 & 30 & AP02325: 75 \\
RAB 2 & 3091.14 & Ile: 16.67 & 6.03 & 0 & 50 & -0.11 & 30 & AP01777: 80.64 \\
RAB 3 & 3313.15 & Ile/Val: 12.9 & 7.76 & 2 & 51 & 0.48 & 30 & AP01990: 74.19 \\
RAB 4 & 3245.94 & Val: 12.9 & 5.53 & 0 & 51 & 0.56 & 30 & AP01062: 77.41 \\
RAB 5 & 3156.35 & Cys: 21.43 & 4.31 & -1 & 46 & 1.00 & 30 & AP00274: 59.37 \\
RAB 6 & 2930.31 & Cys: 21.43 & 3.85 & -1 & 60 & -1.00 & 30 & AP01777: 56.66 \\
RAB 7 & 3197.90 & Cys: 20.69 & 8.38 & 3 & 51 & 3 & 30 & AP02661: 61.29 \\
\hline
\end{tabular}

Moreover, it was ascertained that all the putative anticancer peptides have hydrophobic values that are above $30 \%$, which was the anticipated value for hydrophobicity content of an antimicrobial peptide, and this may pose a significant impact on the binding affinity of the putative AMPs to their cells and receptors [23,24].

\subsection{Predicted 3D Homology Model of the Anti-Cancer Putative AMPs and Slc2a4 by I-TASSER}

The 3D homology model structure of the putative anticancer AMPs and Slc2a4 were analysed using I-TASSER and visualized using PyMol. As showed in (Figure 3) the I-TASSER results indicated that all the model structure of the putative anticancer peptide contained majorly $\beta$-strand which shows that the peptide can modulate and dictate the binding affinity or specificity to their receptor, and it can also help in more promiscuous ligand-protein or protein-protein contacts [25]. Furthermore, I-TASSER result showed that Slc2a4 is made up of 21 helices 359 (70.5\%) $\alpha$-helix, $8(1.6 \%) 3-10$ helix, and 142 $(27.9 \%)$ for others, making 509 residues in total. However, the 3D homology model contradicted the 2D structure, which calls for further investigations. As shown in Table 3, the confidence score (C-score), template modeling score (TM-score) and RMSD of the putative anticancer peptide and the protein 3D homologue model were of good model. The C-score which is the confidence score for evaluating the quality of a predicted model was above the limiting value of -1.5 . Thus, all predicted peptides model was good model because C-score ranges from -5 to 2 [26-28]. Furthermore, the TM-scores were all above the limiting TM-score of $>0.5$ indicating that all the model are of correct topology and a TM-score $<0.17$ indicates a random similarity [26-28]. All the built model has a good and considerable RMSD which ranges from 0.5-0.9 ̊̊ for putative anticancer AMPs and $6.4 \pm 3.9$ for Slc2a4.

Table 3. Quality evaluation scores of the predicted 3D structures by I-TASSER.

\begin{tabular}{cccc}
\hline Putative Anti-Cancer AMPs & C-Score & Exp. TM Score & Exp. RMSD (̊̊) \\
\hline RAB 1 & 0.81 & $0.82 \pm 0.08$ & $0.5 \pm 0.5$ \\
RAB 2 & 0.71 & $0.87 \pm 0.09$ & $0.5 \pm 0.5$ \\
RAB 3 & 0.70 & $0.81 \pm 0.09$ & $0.6 \pm 0.6$ \\
RAB 4 & 0.71 & $0.81 \pm 0.09$ & $0.6 \pm 0.6$ \\
RAB 5 & 0.69 & $0.81 \pm 0.09$ & $0.5 \pm 0.5$ \\
RAB 6 & 0.44 & $0.77 \pm 0.10$ & $0.9 \pm 0.9$ \\
RAB 7 & 0.67 & $0.80 \pm 0.09$ & $0.6 \pm 0.6$ \\
Slc2a4 & 0.42 & $0.77 \pm 0.10$ & $6.4 \pm 3.9$ \\
\hline
\end{tabular}




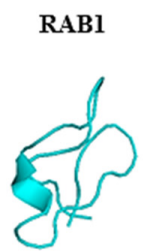

RAB5

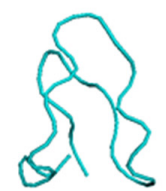

RAB2

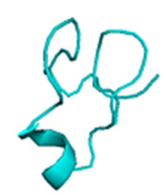

RAB6

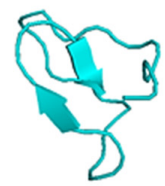

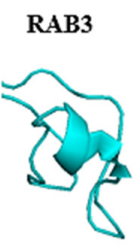

RAB7

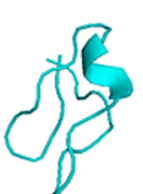

RAB4

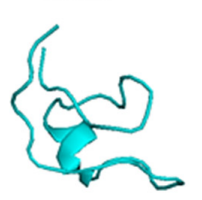

SLC2A4

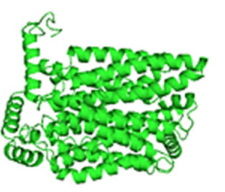

Figure 3. 3D homology model of Slc2a4 and putative anti-cancer AMPs using I-TASSER and visualized using PyMol. The structures in turquoise colour depicted the predicted 3D model of anti-cancer AMPs, and the structure in green colour represented the predicted 3D model of Slc2a4.

\subsection{The 3D Modelled Structure Validation}

Thereafter, the quality of the model was evaluated and validated using PROCHECK, a program that relies on Ramachandran plot for structure verification [29]. As shown in Figure 4, results from the PROCHECK ascertained that the model has $87.4 \%$ residues in the most favoured regions, $10.5 \%$ residues in the additional allowed regions, $1.4 \%$ residues in the generously allowed regions and $0.7 \%$ in the disallowed regions. Therefore, the predicted model is considered to be of high quality because of the percentage distribution of the amino acid residues. The plot analysis is represented in Figure 5. Also, G-factor that provides a measure of how unusual or conversely how usual a given stereochemical property is [30] was also determined using this program. A G-factor of less than -0.5 is unusual and less than -1.0 indicates highly unusual. However, the generate G-factor for this model was -0.43 for dihedral angels, -0.07 for main chain covalent forces and -0.26 overall.

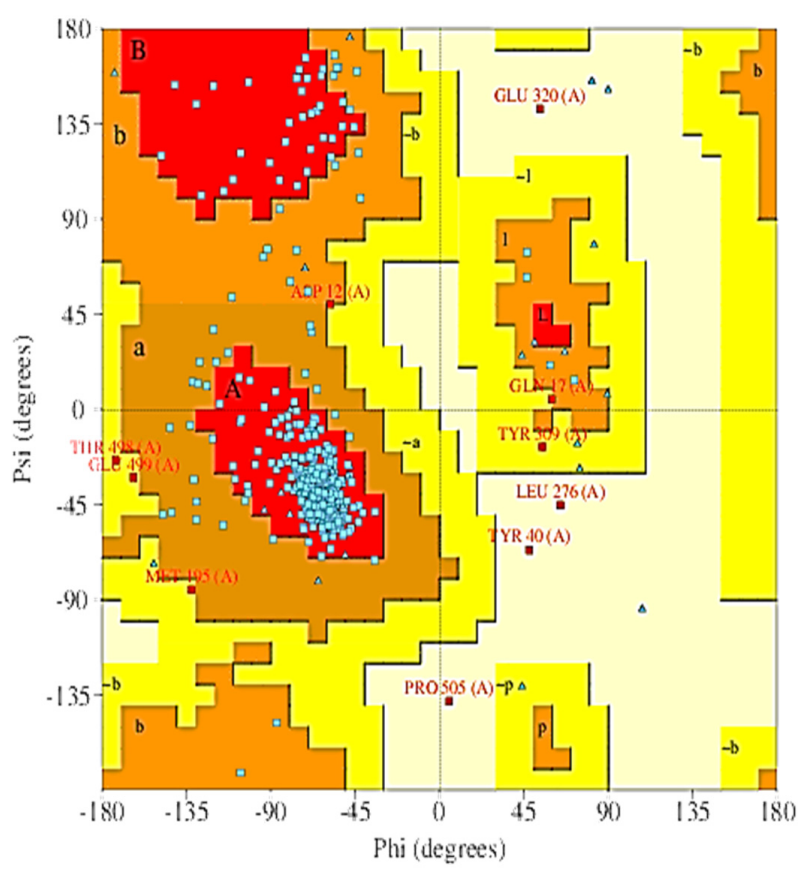

Figure 4. PROCHECK result of modelled Slc2a4 using the generated model from i-TASSER. Residues in most favoured regions (A, B, L), Residues in additional allowed regions $(a, b, 1, p)$ and residues in generously allowed regions $(\sim a, \sim b \sim 1, \sim p)$. 


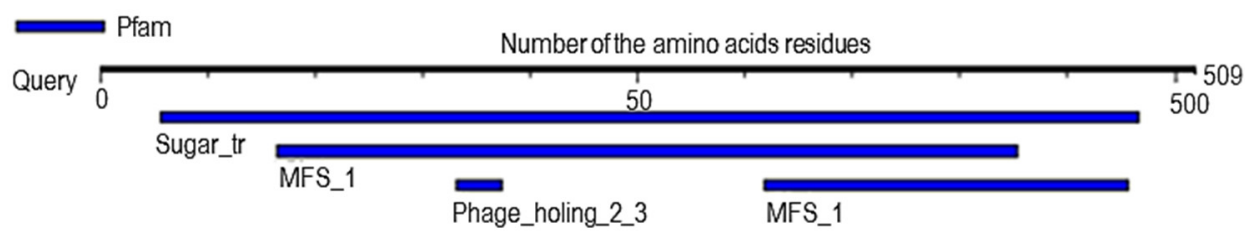

Figure 5. The predicted promising functional motifs present in Slc2a4 generated by motif finder.

\subsection{Analysis of Probable Functional Motifs Presents in Slc2a4 Protein}

As shown in Table 4 and Figure 5, investigation of the functional probable motif ascertained that Slc2a4 contains three functional motifs. Sugar_tr, the first motif, is located at position 28 to 483 in the amino acid sequence with an E-value of $1.8 \times 10^{-152}$, MFS_1 located between position 82 to 427 in the amino acid sequence with E-value of $1.8 \times 10^{-15}$ and position 309 to 478 in the sequence with E-value of 309-478 are the second motif, and lastly, Phage_holin_2_3 located between position 166 to 187 in the amino acid sequence with an E-value of $7.5 \times 10^{-3}$ is the third motif.

Table 4. Motif.

\begin{tabular}{ccccc}
\hline S/N & Pfam ID & Position & E-Value & Description \\
\hline 1 & Sugar_tr & $28-483$ & $1.8 \times 10^{-152}$ & Sugar (and other) transporter \\
2 & MFS_1 & $82-427$ & $1.8 \times 10^{-15}$ & Major Facilitator Superfamily \\
3 & Phage_holin_2_3 & $166-478$ & $4 \times 10^{-3}$ & Bacteriophage holin family HP1 \\
\hline
\end{tabular}

\subsection{The Evaluation and Recognition of Active Sites in Slc2a4 Protein}

Assessment of the protein-ligand binding site prediction for structure-based biological function annotation of $\mathrm{Slc} 2 a 4$ was done using $\mathrm{COACH}$ protein-ligand binding prediction server. The $\mathrm{COACH}$ server predicted all residues that involved in the formation of active site pockets for ligands, which are showed in Table 5 . All the predicted ligands binding site by $\mathrm{COACH}$ server are convincing, because the $\mathrm{C}$-scores ranging between 0 and 1 , and scores close to zero represent random prediction while scores close to 1 indicates more reliable predictions [31].

Table 5. Predicted ligand binding sites in the Slc2a4 using the homologue model generated by I-TASSER.

\begin{tabular}{|c|c|c|c|}
\hline $\begin{array}{l}\text { Name of } \\
\text { Server }\end{array}$ & Name of Ligand & Residue Number & C-Score \\
\hline \multirow{10}{*}{$\begin{array}{l}\text { COACH } \\
\text { SERVER }\end{array}$} & Maltose (MAL) & $\begin{array}{c}42,46,177,180,181,184,298,299,304 \\
333,395,396,404,431\end{array}$ & 0.19 \\
\hline & Cytochalasin B (5RH) & $\begin{array}{c}96,153,177,180,298,304,400,424,427 \\
428,431\end{array}$ & 0.10 \\
\hline & Cholesterol hemisuccinate (Y01) & $97,275,292,296,300,426,429,434$ & 0.09 \\
\hline & $\begin{array}{l}\text { (2 \{S\})-3-(2-bromophenyl)-2-[2-(4- } \\
\text { methoxyphenyl)ethanoylamino]- }\{\mathrm{N}\}-[(1 \sim\{\mathrm{S}\})-1- \\
\text { phenylethyl] propenamide }(5 \mathrm{RF})\end{array}$ & $\begin{array}{c}38,96,153,176,298,395,396,420,424 \\
427,428\end{array}$ & 0.05 \\
\hline & Octyl Glucose Neopentyl Glycol (37X) & $22,23,26,218,219,222,246$ & 0.03 \\
\hline & (2S)-2,3Dihydroxypropyl (7Z)-pentadec-7-enoate (78M) & $90,91,93,94,142,426,427$ & 0.02 \\
\hline & Octyl Glucose Neopentyl Glycol (37X) & $94,97,98,101,118,143$ & 0.02 \\
\hline & methyl- $\alpha$-D-glucopyranoside (GYP) & $296,430,433,434,452,453,456,457$ & 0.02 \\
\hline & N-[(1R)-1-phosphonoethyl]-L-alaninamide (AFS) & $39,43,177,180,181,184,329,333$ & 0.02 \\
\hline & M-cresol (CRS) & 306,309 & - \\
\hline
\end{tabular}


Table 5. Cont.

\begin{tabular}{|c|c|c|c|}
\hline $\begin{array}{l}\text { Name of } \\
\text { Server }\end{array}$ & Name of Ligand & Residue Number & C-Score \\
\hline \multirow{4}{*}{$\begin{array}{l}\text { TM-Site } \\
\text { Results }\end{array}$} & $\begin{array}{c}\text { Maltose (MAL) (2), } \beta \text {-D-glucose (BGC) (2), } \\
\text { 6-bromo-6-deoxy- } \beta \text {-D-glucopyranose (6BG) (1) }\end{array}$ & $\begin{array}{c}42,46,177,180,181,184,298,299,304 \\
333,395,396,404,431\end{array}$ & 0.4 \\
\hline & $\begin{array}{l}\text { 5RH }(1),(2 \sim\{S\})-3-(2-\text { bromophenyl)-2-[2-(4- } \\
\text { methoxyphenyl)ethanoylamino]- }\{\mathrm{N}\}-[(1 \sim\{\mathrm{S}\})-1- \\
\text { phenylethyl] propenamide }(5 \mathrm{RF})(1), \\
(2 \sim\{\mathrm{S}\})-3-(4-\text {-fluorophenyl)-2-[2-(3- } \\
\text { hydroxyphenyl)ethanoylamino]- }\{\mathrm{N}\}-[(1 \sim\{\mathrm{S}\})-1- \\
\text { phenylethyl]propenamide(5RE(1) }\end{array}$ & $\begin{array}{c}96,153,177,180,298,304,400,424,427 \\
428,431\end{array}$ & 0.2 \\
\hline & Cholesterol hemisuccinate (Y01) (2) & $97,275,292,296,300,426,429,434$ & 0.1 \\
\hline & Octyl Glucose Neopentyl Glycol (37X) (1) & $22,23,26,218,219,222,246$ & 0.1 \\
\hline \multirow{5}{*}{$\begin{array}{l}\text { S-Site } \\
\text { Results }\end{array}$} & $\begin{array}{l}\text { 2'-deoxycytidine-5'-monophosphate (DCM) (1), } \\
2^{\prime} \text {-deoxyuridine } 5^{\prime} \text {-monophosphate (UMP) (1) }\end{array}$ & 349,350 & 0.16 \\
\hline & M-cresol (CRS) (1), adenosine-5'-phosphosulfate (ADX) (1) & $304,306,309$ & 0.15 \\
\hline & magnesium ion $\left(\mathrm{Mg}^{2+}\right)(1)$, calcium ion $\left(\mathrm{Ca}^{2+}\right)(1)$ & $224,225,226,228$ & 0.15 \\
\hline & magnesium ion $\left(\mathrm{Mg}^{2+}\right)(1)$ & 162,165 & 0.14 \\
\hline & $\begin{array}{l}\text { L- } \alpha \text {-glycerophosphorylethanol amine (GPE) (1), } \\
\text { Fe (II) ion }\left(\mathrm{Fe}^{2+}\right)(1)\end{array}$ & $239,299,302,303,400,409$ & 0.14 \\
\hline
\end{tabular}

\subsection{The Protein-Peptide Interaction between the Putative Anticancer AMPs and Slc2a4 Protein}

The docking results of the structural complex between the putative anticancer AMPs and the Slc2a4 protein were downloaded as PDB files, and they were all visualized using PyMol software. This is shown in Figure 6 in which the receptor (Slc2a4) as green surface and the ligand (AMPs) are shown in turquois blue.

\section{Slc2a4-RAB1}
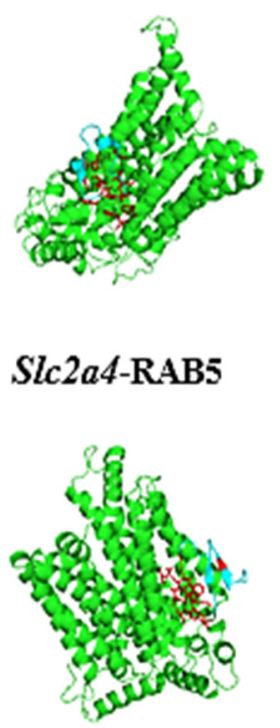

\section{Slc2a4-RAB2}

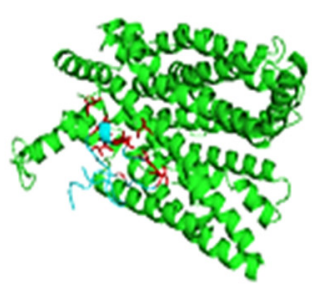

Slc2a4-RAB6

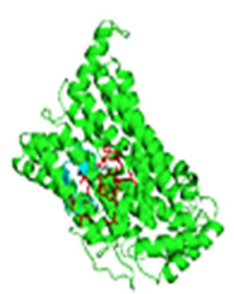

Slc2a4-RAB3

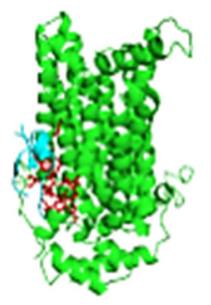

Slc2a4-RAB7

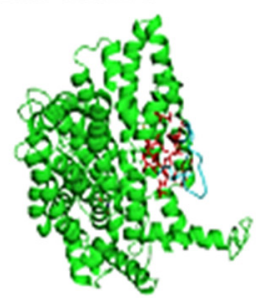

\section{Slc2a4-RAB4}

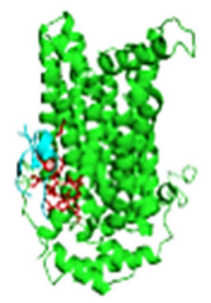

Figure 6. Interaction of putative anti-cancer AMPs with Slc2a4. The turquoise colours depicted the anti-cancer AMPs (RABs), green colours represented the Slc2a4 and red colours shown the binding site.

\section{Disccusion}

Solute Carrier Family 2 Member 4 (Slc2a4), otherwise known as GLUT4, is a member of the glucose transporter family [32]. Slc2a4 can serve as a potential biomarker for diverse kinds of malignant tumours such as; breast cancer, lung carcinoma, gastric cancer, and endometrial carcinoma [33,34]. 
Although, the exact mode of action between Slc2a4 and cancer formation, progression and metastasis as not been fully understood. The uncontrolled growth and proliferation of cancer cells need a constant supply of metabolic energy, and glycolysis is one of the main biochemical process that characterised tumour cells, and the glycolytic breakdown of glucose is initiated by the transport of glucose, a rate-limiting process which is mediated by GLUT [35]. Moreover, an increase in GLUT upregulation in malignant cells has been linked with the overexpression of GLUT proteins, which supply steady metabolic energy to cancer cells [35]. Numerous research studies have hypothesis several ways in which Slc2a4 results in cancer progression and metastasis one of which is the regulation of TRIM24-DDX58 axis that promote head and neck cancer (HNCC) metastasis [36]. Also, Slc2a4 has been shown to participates actively in glucose uptake in androgen-insensitive than in androgen-sensitive in prostates cancer cells [35]. To the best of our knowledge, this study is novel and significant as it's the first report on AMPs as inhibitors of Slc2a4 for the treatment of cancer. Therefore, we have in this study analysed the Slc2a4 protein sequence and designed seven putative anticancer AMPs as well as studied the protein-peptide interaction between the putative AMPs and the Slc2a4 protein using several types of highly precise bioinformatics tools.

In Slc2a4, cysteine and tryptophan contributed less stability and folding state to the protein, due to the presents of their lesser amount in the protein. Cysteine contain reactive sulfhydryl group (-SH) that has ability to oxidize and form a disulphide bond (-S-S-) to a second cysteine [18]. Also, tryptophan contains an aromatic side chain that makes it able to interact with the membrane interface, stabilize the membrane protein, and contribute to hydrophobic mismatch response [37]. Thus, the stability of the Slc2a4 protein might be due to the burial of polar groups and their hydrogen bonds [38] as well as the net charge and the ionization state of the individual amino acid residues [39]. The stability of Slc2a4 protein may also be due to the presence of thousands of noncovalent bonds between amino acids and the availability of chemical forces between the protein and its immediate environment [40].

Hence, there are several ongoing searches for novel drugs that can serves as alternative medications in the treatment and management of cancer. Antimicrobial peptides have gained noticeable quality as possible sources of novel medicine in the treatment and control of diseases. This significant important of therapeutic applications of antimicrobials peptide in the treatment and control of diseases may be due to their size as well as their diverse activities and properties triggered toward diseases [17]. In this study, seven (7) putative anticancer AMPs were generated, with the majority having a Boman index less than or equal to one which indicates that the peptides will make good antimicrobial activity with minimal adverse effects. Also, the zero-net charge of some of the peptides may influences the binding capacity of those peptide to bind to the receptor. It has been shown that AMPs with Boman index less than zero (0) only possess antibacterial activity while AMPs with Boman index greater than zero have multifunctional hormone-like activities [41]. The 3D structure of the seven putative anticancer AMPs possess $\beta$-strand which can help in the folding complementation, insertion and intermolecular interactions between the hydrogen-bonding edges of $\beta$-sheets. This type of hydrogen bond interaction has been shown to contribute majorly to the fundamental form of biomolecular recognition, as well as, involvement in protein quaternary structure, protein and peptide aggregation and protein-protein interactions [42]. Therefore, the presence of $\beta$-sheet in AMPs make them to interact with another biomolecule; this biological importance exhibited by AMPs make it to have significant impact as potential targets for intervention in diseases. Furthermore, the 3D structure of Slc2a4 protein was determined and both the 3D structure of the modelled putative anticancer AMPs and the Slc2a4 protein are of good quality; when considering their C-score, TM-score and RMSD value. Likewise, the reliability and quality of the modelled Slc2a4 protein was further evaluated using PROCHECK, which revealed that the protein 3D modelled structure is deemed to be of high quality because of the percentage distribution of the amino acid residues in the most favoured, allowed, disallowed, and generously allowed regions. Thereafter, three major probable functional motifs were predicted, and several ligands active sites presents in the Slc2a4 protein was recognised. 
Finally, the generated anticancer putative AMPs were used in this study to inhibits Slc2a4; an important protein that responsible for energy productions during angiogenesis in tumour formation and this can serve as another novel therapeutic agent for the treatment and management of cancer.

In this study, online protein-protein interaction tools PatchDock and FireDock server were used as docking tool for our putative anticancer AMPs (RAB1- RAB7) with Slc2a4 protein. The generated 3D structure of the seven-novel anticancer putative AMPs serves as ligands, and each ligand were docked against the 3D structure of Slc2a4 protein respectively. The docking runs for each of the parameter (AMP and Slc2a4) were done using PatchDock server and all the results generated by this server showed a very high binding affinity (score) which is greater than 8741 [43]. This indicates good binding solution for all the putative anticancer AMPs with RAB 1 having the highest binding affinity (score) as shown in Table 6. Furthermore, the generated results were refined using FireDock server, and all the results strongly supports the PatchDock score with a very good binding energy that is; the global energy in which RAB 5, RAB 4, and RAB 3 has less binding energy with $-68.18,-55.01$ and $-53.99 \mathrm{kcal} / \mathrm{mol}$, respectively. Altogether, the results reported in this study can usefully be employed for the rational design of novel, selective and potent Slc2a4 inhibitors in the search for novel anti-cancer therapy with high efficacy.

Table 6. PatchDock and FireDock results for each AMPs, with the binding affinity and binding energy for the solution in $\mathrm{kcal} / \mathrm{mol}$.

\begin{tabular}{ccc}
\hline Molecule (AMPs) & Binding Affinity for Geometry Scores & $\begin{array}{c}\text { Global Energy (Binding Energy } \\
\text { for the Solution) (kcal/mol) }\end{array}$ \\
\hline RAB 1 & 12,392 & -39.13 \\
RAB 2 & 11,528 & -36.74 \\
RAB 3 & 10,154 & -53.99 \\
RAB 4 & 10,768 & -55.01 \\
RAB 5 & 11,146 & -68.19 \\
RAB 6 & 10,556 & -25.85 \\
RAB 7 & 11,558 & -36.88 \\
\hline
\end{tabular}

\section{Materials and Methods}

\subsection{Sequence Retrieval and Physicochemical Parameters Analysis of Slc2a4}

The amino acid sequences of the protein Slc2a4 were retrieved from the Universe Protein Resource (UniProt) (http://www.uniprot.org/), a database containing 80 million sequences [44]. The physicochemical parameters of the protein were generated from the ProtParam tools (http:/ / web.expasy.org/protparam/) using the ExPAsy server. They reveal vital information about the protein structure and function. Knowing that the physicochemical parameters of a protein is of paramount importance, the following parameters were computed by the ProtParam tool: molecular weight, extinction coefficient, amino acid composition [45], estimated half-life and grand average of hydropathicity (GRAVY), theoretical pI [46], as well as aliphatic index [47] and instability index.

\subsection{Secondary Structure Prediction}

Prediction of the secondary structure ( $\alpha$-helices, $\beta$-sheets and random coils) of Slc2a4 was done using PsiPred secondary structure prediction server (http://bioinf.cs.ucl.ac.uk/psipred). PSIPRED is a highly accurate tool for protein secondary structure prediction from its primary sequence, which integrates two feed-forward neural networks that perform an analysis on output obtained from PSI-BLAST (Position Specific Iterated-BLAST) [48]. 


\subsection{Experimentally Validated Antimicrobial Peptides (AMPs) Data Assessment}

Several experimentally validated anticancer antimicrobial peptides (AMPs) originating from plants were retrieved from different antimicrobial peptide databases that include Collection of Antimicrobial Peptides (CAMP) (http://www.camp.bicnirrh.res.in) and Data Repository of Antimicrobial Peptides (DRAMP) (http:/ / dramp.cpu-bioinfor.org). Subsequently, the retrieved data was organised and curated to verify the authenticity of the experimentally validated anticancer AMPs. Thereafter, Cluster Database at High Identity with Tolerance (CD HIT) (http:/ / www.bioinformatics. $\mathrm{org} / \mathrm{cd}$-hit) was used to remove the experimentally validated anticancer AMPs which are in duplicate.

\subsection{Construction of AMPs Profile Using Hidden Markov Models}

The list of the generated plants experimentally validated anticancer AMPs was divided into two portions, in which one-quarter of the retrieved data was utilised as the testing set while the three-quarters of the retrieved data was used as the training set. The Hidden Markov Models (HMMER) algorithm version 2.3.2 was used to create HMMs profile by utilizing training sets. Afterward, the testing set was used to ascertain the strength of the profile. The HMMER multiple modules was used in the construction of the AMPs profile.

Firstly, using clustalW as multiple alignment tool, a multiple sequence alignment was done for the sequences in the training set using the command line:

Clustalw-align-output=gcg-case=upper-seqnos=off-outorder=aligned-infile=family. fasta

The result was saved as msf (gcg) format (family.msf). This aligned sequence was used for the second step, this step shows the common motifs within the model and it was achieved by inputting the command line:

hmmbuild file.hmm family.msf

The sensitivity of the new profile HMM was enhanced by calibrating it using the command line:

hmmcalibrate family.hmm

The resulting profile 'family.hmm' was employed in evaluating the performance of the profile by scanning it against AMPs in the testing set. The E-value threshold was set to $1 \%$ or $0.01 \%$ and the scanning was achieved using the command line:

hmmsearch -E 1e-2 family.hmm family(test set).fasta > resultname.txt

Discovery of Novel Putative Anticancer Amps from Genome Sequences

The ENSEMBL server (http://www.ensembl.org/index.html) and UNIPROT database (http:/ / www.uniprot.org/) were visited to retrieve at least a thousand genome sequences in FASTA format. In order to identify the putative anti-cancer AMPs, the constructed profile was queried against all the genome sequences with an E-value cut-off of 0.01 . This was achieved by utilizing the hmm search module of HMMER and the command line used is:

hmmsearch -E 1e-2 family.hmm family(test set).fasta > resultname.txt 
All identified peptides were considered to be putative anti-cancer AMPs.

\subsection{Determination of the Physicochemical Parameters of the Putative Anti-Anticancer AMPs}

Several physicochemical parameters of the putative anticancer AMPs were determined using the prediction interface of APD (http://aps.unmc.edu/AP/design/design_improve.php) and Bactibase (http://bactibase.pfba-lab-tun.org/physicochem), respectively. Some of the properties include molecular weight, the most abundant amino acids, net charge, isoelectric point, total hydrophobic ratio, Half-life, Boman index, and sequence similarity with other molecules and percentage (\%).

\subsection{Homology Modelling of the Three-Dimensional Structure of Slc2a4 and Putative Anti-Cancer AMPs}

The 3D structure prediction of the seven putative anticancer AMPs and Slc2a4 protein was done using I-TASSER server (http:/ / zhanglab.ccmb.med.umich.edu/I-TASSER/) online database, the best results was obtained based on their E-values. The amino acid sequences were submitted to the online server of the I-TASSER and the result generated in PDB format was visualized using PyMol [49]. In predicting the 3D structure of SLC2A4, the protein with PDB ID: 4pypA (Crystal structure of the human glucose transporter GLUT1) was utilized as template for de-novo modeling of the SLC2A4 protein, out of the 10 top templates chosen from the LOMETS threading programs. This is based on the highest significance in the threading alignments of the template with the query protein, which is measured by the Z-score; the difference between the average and raw scores in the unit of standard deviation [50].

\subsection{Evaluation of the Generated Model}

The stereochemical quality of the generated model was checked using a program called PROCHECK [51]. It verifies the quality of model by computing several parameters such as side chain conformations of protein structures as a function of atomic resolution, lengths, geometry of the hydrogen bonds, and angles and planarity of the peptide bonds [29].

\subsection{Investigation of the Probable Functional Motif}

MOTIF Finder (http://www.genome.jp/tools/motif/) an online search engine was used to ascertain the probable motifs present in the protein by analysis the amino acid sequences. A statistical expect value (E-value) cut off score is given because it shows the significance of the hit.

\subsection{Active Site Analysis and Residues Recognition}

The prediction of active site and possible ligand binding residues of Slc2a4 was generated using $\mathrm{COACH}$ protein-ligand binding prediction server, a meta-server approach to protein-ligand binding site prediction (http://zhanglab.ccmb.med.umich.edu/COACH/). The complementary ligand binding site was predicted using $\mathrm{COACH}$ by matching the Slc2a4 I-TASSER generated model with protein in the BioLiP protein function database. Also, the functional templates are detected and ranked by $\mathrm{COACH}$ using composite scoring function that is based on structure and sequence profile comparisons [26].

\subsection{In Silico Protein-Protein Interaction Study}

Having identified our putative anti-cancer AMPs, we decided to use PATCH Dock, an online protein-protein interaction server (https://bioinfo3d.cs.tau.ac.il/PatchDock), for performing the molecular docking. This server depends on shape complementarity of soft molecular surfaces to generate the best starting candidate solution [52]. The default clustering RMSD 4.0 A was used and the complex type was chosen to be at protein-small ligand. The Connolly dot surface representation of the molecules into different component such as convex, concave, and flat patches were generated through PatchDock algorithm. Thereafter, the complementary patches were harmonized to form transformation 
candidates, which were later refined using FireDock server (http:/ /bioinfo3d.cs.tau.ac.il/FireDock/), an online tool that optimised, refined, reshuffled, and rescored the side chains interface of the top 10 candidate solutions. It also amends the orientation of the relative molecules by confining the flexibility to the side-chains of the interacting surface and allow the movements of small rigid-body [53]. Therefore, the interactions and binding observed in the docked conformations in PDB format were visually using the PyMol software [49].

Acknowledgments: Research reported in this article was supported by the University of Zululand Research Committee. We like to thank Chinaza Uleanya for proofreading the final draft.

Author Contributions: Raphael Taiwo Aruleba, Tayo Alex Adekiya and Babatunji Emmanuel Oyinloye conceived and designed the experiments. They also performed the experiments and analyzed the data. Abidemi Paul Kappo gave intellectual input regarding the experimental design and draft paper. All authors wrote the final copy of the paper.

Conflicts of Interest: The authors declare no conflict of interest.

\section{References}

1. Cao, B.; Bray, F.; Beltrán-Sánchez, H.; Ginsburg, O.; Soneji, S.; Soerjomataram, I. Benchmarking life expectancy and cancer mortality: Global comparison with cardiovascular disease 1981-2010. BMJ 2017, 357, j2765. [CrossRef] [PubMed]

2. Kalyanaraman, B. Teaching the basics of cancer metabolism: Developing antitumor strategies by exploiting the differences between normal and cancer cell metabolism. Redox Biol. 2017, 12, 833-842. [CrossRef] [PubMed]

3. Pande, A.N.; Biswas, S.; Reddy, N.D.; Jayashree, B.S.; Kumar, N.; Rao, C.M. In vitro and in vivo anticancer studies of 2'-hydroxy chalcone derivatives exhibit apoptosis in colon cancer cells by HDAC inhibition and cell cycle arrest. EXCLI J. 2017, 16, 448-463. [PubMed]

4. Anand, P.; Kunnumakara, A.B.; Sundaram, C.; Harikumar, K.B.; Tharakan, S.T.; Lai, O.S.; Sung, B.; Aggarwal, B.B. Cancer is a Preventable Disease that Requires Major Lifestyle Changes. Pharm. Res. 2008, 25, 2097-2116. [CrossRef] [PubMed]

5. Batra, S.; Adekola, K.U.; Rosen, S.T.; Shanmugam, M. Cancer metabolism as a therapeutic target. Oncology 2013, 27, 460-467. [PubMed]

6. Seyfried, T.N. Cancer as a mitochondrial metabolic disease. Front. Cell Dev. Biol. 2015, 3, 43. [CrossRef] [PubMed]

7. Torre, L.A.; Bray, F.; Siegel, R.L.; Ferlay, J.; Lortet-Tieulent, J.; Jemal, A. Global cancer statistics, 2012. CA Cancer J. Clin. 2015, 65, 87-108. [CrossRef] [PubMed]

8. Ferlay, J.; Soerjomataram, I.; Dikshit, R.; Eser, S.; Mathers, C.; Rebelo, M.; Parkin, D.M.; Forman, D.; Bray, F. Cancer incidence and mortality worldwide: Sources, methods and major patterns in GLOBOCAN 2012. Int. J. Cancer 2015, 136, E359-E386. [CrossRef] [PubMed]

9. Stewart, B.W.; Bray, F.; Forman, D.; Ohgaki, H.; Straif, K.; Ullrich, A.; Wild, C.P. Cancer prevention as part of precision medicine: 'Plenty to be done'. Carcinogenesis 2016, 37, 2-9. [CrossRef] [PubMed]

10. Liang, X.J.; Chen, C.; Zhao, Y.; Wang, P.C. Circumventing tumorresistance to chemotherapy by nanotechnology Methods. Mol. Biol. 2010, 596, 467-488.

11. Desai, A.G.; Qazi, G.N.; Ganju, R.K.; El-Tamer, M.; Singh, J.; Saxena, A.K.; Bedi, Y.S.; Taneja, S.C.; Bhat, H.K. Medicinal plants and cancer chemoprevention. Curr. Drug Metab. 2008, 9, 581-591. [CrossRef] [PubMed]

12. Mishra, R.K.; Wei, C.; Hresko, R.C.; Bajpai, R.; Heitmeier, M.; Matulis, S.M.; Nooka, A.K.; Rosen, S.T.; Hruz, P.W.; Schiltz, G.E.; et al. In silico modeling-based identification of glucose transporter 4 (GLUT4)-selective inhibitors for cancer therapy. J. Biol. Chem. 2015, 290, 1441-1453. [CrossRef] [PubMed]

13. Schwartzenberg-Bar-Yoseph, F.; Armoni, M.; Karnieli, E. The tumor suppressor p53 down-regulates glucose transporters GLUT1 and GLUT4 gene expression. Cancer Res. 2004, 64, 2627-2633. [CrossRef] [PubMed]

14. Zhao, Y.; Butler, E.B.; Tan, M. Targeting cellular metabolism to improve cancer therapeutics. Cell Death Dis. 2013, 4, e532. [CrossRef] [PubMed] 
15. Tarazona-Santos, E.; Fabbri, C.; Yeager, M.; Magalhães, W.C.S.; Burdett, L.; Crenshaw, A.; Pettener, D.; Chanock, S.J. Diversity in the glucose transporter-4 gene (SLC2A4) in Humans reflects the action of natural selection along the old-world primates evolution. PLOS ONE 2010, 5, e9827. [CrossRef] [PubMed]

16. Calvo, M.B.; Figueroa, A.; Pulido, E.G.; Campelo, R.G.; Aparicio, L.A. Potential role of sugar transporters in cancer and their relationship with anticancer therapy. Int. J. Endocrinol. 2010, 2010, 205357. [CrossRef] [PubMed]

17. Oyinloye, B.; Adenowo, F.; Gxaba, N.; Kappo, A. The promise of antimicrobial peptides for treatment of human schistosomiasis. Curr. Drug Targets 2014, 15, 852-859. [CrossRef] [PubMed]

18. Lodish, H.; Berk, A.; Zipursky, S.L.; Matsudaira, P.; Baltimore, D.; Darnell, J. Molecular Cell Biology, 4th ed.; W.H. Freeman: New York, NY, USA, 2000.

19. Michelle Furtado, L.; Poon, V.; Klip, A. GLUT4 activation: Thoughts on possible mechanisms. Acta Physiol. Scand. 2003, 178, 287-296. [CrossRef] [PubMed]

20. Huang, S.; Czech, M.P. The GLUT4 glucose transporter. Cell Metab. 2007, 5, 237-252. [CrossRef] [PubMed]

21. Stuart, C.A.; Yin, D.; Howell, M.E.; Dykes, R.J.; Laffan, J.J.; Ferrando, A.A. Hexose transporter mRNAs for GLUT $_{4}$, GLUT $_{5}$, and GLUT 12 predominate in human muscle. Am. J. Physiol. Endocrinol. Metab. 2006, 291, E1067-E1073. [CrossRef] [PubMed]

22. Xiong, J. Essential Bioinformatics; Cambridge University Press: New York, NY, USA, 2000; p. 352.

23. Hancock, R.E.; Sahl, H.G. Antimicrobial and host-defense peptides as new anti-infective therapeutic strategies. Nat. Biotechnol. 2006, 24, 1551-1557. [CrossRef] [PubMed]

24. Giuliani, A.; Pirri, G.; Nicoletto, S. Antimicrobial peptides: An overview of a promising class of therapeutics. Open Life Sci. 2007, 2, 1-33. [CrossRef]

25. Remaut, H.; Waksman, G. Protein-protein interaction through $\beta$-strand addition. Trends Biochem. Sci. 2006, 31, 436-444. [CrossRef] [PubMed]

26. Yang, J.; Zhang, Y. Protein Structure and Function Prediction Using I-TASSER. In Current Protocols in Bioinformatics; John Wiley and Sons, Inc.: Hoboken, NJ, USA, 2015; pp. 5-8.

27. Roy, A.; Kucukural, A.; Zhang, Y. I-TASSER: A unified platform for automated protein structure and function prediction. Nat. Protoc. 2010, 5, 725-738. [CrossRef] [PubMed]

28. Zhang, Y. I-TASSER server for protein 3D structure prediction. BMC Bioinform. 2008, 9, 40. [CrossRef] [PubMed]

29. Chhabra, G.; Sharma, P.; Anant, A.; Deshmukh, S.; Kaushik, H.; Gopal, K.; Srivastava, N.; Sharma, N.; Garg, L.C. Identification and modeling of a drug target for Clostridium perfringens SM101. Bioinformation 2010, 4, 278-289. [CrossRef] [PubMed]

30. Laskowski, R.A.; Rullmann, J.A.C.; MacArthur, M.W.; Kaptein, R.; Thornton, J.M. AQUA and PROCHECK-NMR: Programs for checking the quality of protein structures solved by NMR. J. Biomol. NMR 1996, 8, 477-486. [CrossRef] [PubMed]

31. Roche, D.B.; Brackenridge, D.A.; McGuffin, L.J. Proteins and their interacting partners: An introduction to protein-ligand binding site prediction methods. Int. J. Mol. Sci. 2015, 16, 29829-29842. [CrossRef] [PubMed]

32. Aparicio, L.M.A.; Villaamil, V.M.; Calvo, M.B.; Rubira, L.V.; Rois, J.M.; Valladares-Ayerbes, M.; Campelo, R.G.; Bolós, M.; Pulido, E.G. Glucose transporter expression and the potential role of fructose in renal cell carcinoma: A correlation with pathological parameters. Mol. Med. Rep. 2010, 3, 575-580. [CrossRef] [PubMed]

33. Ito, T.; Noguchi, Y.; Satoh, S.; Hayashi, H.; Inayama, Y.; Kitamura, H. Expression of facilitative glucose transporter isoforms in lung carcinomas: Its relation to histologic type, differentiation grade, and tumor stage. Mod. Pathol. 1998, 11, 437-443. [PubMed]

34. Garrido, P.; Osorio, F.G.; Morán, J.; Cabello, E.; Alonso, A.; Freije, J.M.; González, C. Loss of GLUT4 induces metabolic reprogramming and impairs viability of breast cancer cells. J. Cell. Physiol. 2015, 230, 191-198. [CrossRef] [PubMed]

35. Chen, C.; Shen, H.; Zhang, L.G.; Liu, J.; Cao, X.G.; Yao, A.L.; Kang, S.S.; Gao, W.X.; Han, H.; Cao, F.H.; et al. Construction and analysis of protein-protein interaction networks based on proteomics data of prostate cancer. Int. J. Mol. Med. 2016, 37, 1576-1586. [CrossRef] [PubMed]

36. Chang, Y.C.; Hsiao, M. Solute Carrier Family 2 Member 4 Regulates TRIM24-DDX58 Axis to Promote Head and Neck Cancer Metastasis. FASEB J. 2017, 31 (Suppl. 808.5), 808. 
37. De Jesus, A.J.; Allen, T.W. The determinants of hydrophobic mismatch response for transmembrane helices. Biochim. Biophys. Acta 2013, 1828, 851-863. [CrossRef] [PubMed]

38. Pace, C.N.; Fu, H.; Fryar, K.L.; Landua, J.; Trevino, S.R.; Schell, D.; Grimsley, G.R. Contribution of hydrogen bonds to protein stability. Protein Sci. 2014, 23, 652-661. [CrossRef] [PubMed]

39. Pace, C.N.; Grimsley, G.R.; Scholtz, J.M. Protein ionizable groups: $\mathrm{pK}$ values and their contribution to protein stability and solubility. J. Biol. Chem. 2009, 284, 13285-13289. [CrossRef] [PubMed]

40. Petsko, G.A.; Ringe, D. Protein Structure and Function; New Science Press: London, UK, 2004.

41. Boman, H.G. Antibacterial peptides: Basic facts and emerging concepts. J. Intern. Med. 2003, 254, $197-215$. [CrossRef] [PubMed]

42. Nowick, J.S. Exploring $\beta$-sheet structure and interactions with chemical model systems. Acc. Chem. Res. 2008, 41, 1319-1330. [CrossRef] [PubMed]

43. Gujjula, K.R. Prediction and Comparison of HIV-1 Protease Inhibitor Binding Energies by Various Molecular Docking Methods. Ph.D. Dissertation, National Institute of Technology Rourkela, Orissa, India, 2008.

44. The UniProt Consortium. UniProt: A hub for protein information. Nucleic Acids Res. 2015, 43, D204-D212.

45. Gill, S.C.; von Hippel, P.H. Calculation of protein extinction coefficients from amino acid sequence data. Anal. Biochem. 1989, 182, 319-326. [CrossRef]

46. Kyte, J.; Doolittle, R.F. A simple method for displaying the hydropathic character of a protein. J. Mol. Biol. 1982, 157, 105-132. [CrossRef]

47. Ikai, A.J. Thermostability and aliphatic index of globular proteins. J. Biochem. 1980, 88, 1895-1898. [PubMed]

48. McGuffin, L.J.; Bryson, K.; Jones, D.T. The PSIPRED protein structure prediction server. Bioinformatics 2000, 16, 404-405. [CrossRef] [PubMed]

49. DeLano, W.L. The PyMOL Molecular Graphics System. 2002. Available online: http:/ / pymol.Org (accessed on 25 September 2016).

50. Yang, J.; Yan, R.; Roy, A.; Xu, D.; Poisson, J.; Zhang, Y. The I-TASSER Suite: Protein structure and function prediction. Nat. Methods 2015, 12,7-8. [CrossRef] [PubMed]

51. Laskowski, R.A.; MacArthur, M.W.; Moss, D.S.; Thornton, J.M. PROCHECK: A program to check the stereochemical quality of protein structures. J. Appl. Cryst. 1993, 26, 283-291. [CrossRef]

52. Schneidman-Duhovny, D.; Inbar, Y.; Nussinov, R.; Wolfson, H.J. PatchDock and SymmDock: servers for rigid and symmetric docking. Nucleic Acids Res. 2005, 33, W363-W367. [CrossRef] [PubMed]

53. Duhovny, D.; Nussinov, R.; Wolfson, H.J. Efficient unbound docking of rigid molecules. Lect. Notes Comput. Sci. 2002, 2452, 185-200. 